International Journal of Uncertainty, Fuzziness and Knowledge-Based Systems (c) World Scientific Publishing Company

\title{
Uncertainty-Aware Dissimilarity Measures for Interval-Valued Fuzzy Sets
}

\author{
Emilio Torres-Manzanera \\ Department of Statistics, University of Oviedo, Luis Moya 261, \\ Gijón, 33203, Spain \\ torres@uniovi.es \\ Pavol Král' \\ Faculty of Economy, Matej Bel University, Tajovského 10, \\ Banská Bystric, 975 90, Slovak Republic \\ pavol.kral@umb.sk \\ Vladimír Janiš \\ Faculty of Nature Science, Matej Bel University, Tajovského 40 \\ Banská Bystrica, 974 01, Slovak Republic \\ vladimir.janis@umb.sk \\ Susana Montes \\ Department of Statistics, University of Oviedo, Luis Ortiz Berrocal \\ Gijón, 33203, Spain \\ montes@uniovi.es \\ Received (received date) \\ Revised (revised date)
}

\begin{abstract}
Dissimilarities are a very usual way to compare two fuzzy sets and also two intervalvalued fuzzy sets. In both cases, the dissimilarity between two sets is a number. In this work, we introduce a generalization of the notion of dissimilarity for interval-valued fuzzy sets such that it assumes values on the set of subintervals instead of the set of numbers. This seems to be more realistic taking into account the available information. We also investigate its relationship with the classical notions of dissimilarity between fuzzy sets and we obtain that the new class is richer than the existing one.
\end{abstract}

Keywords: Dissimilarity, Interval-valued sets, Order on interval-valued fuzzy sets

\section{Introduction}

There are several ways how a grade of mutual difference between two fuzzy sets can be expressed. Families of such estimations are known as similarities (with their dual functions dissimilarities), divergences or similitudes (dual function dissimilitudes). The motivation for our work comes from similarities, but also other measures of difference could be used. 

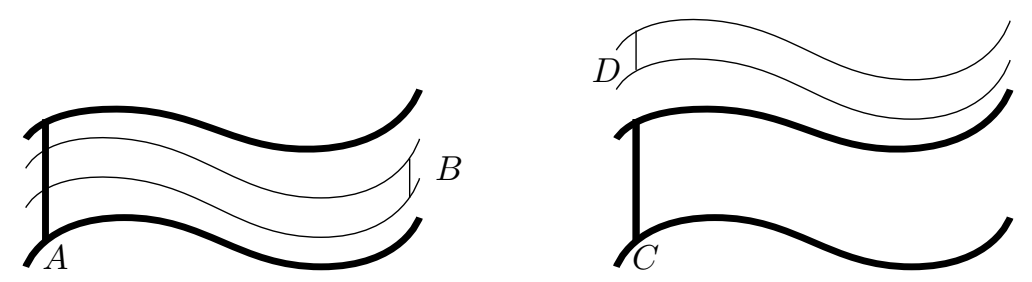

Fig. 1. Two pairs of interval-valued fuzzy sets

Currently several measures of comparison between fuzzy sets have been studied. In 1996, Bouchon-Meunier ${ }^{1}$ defined a general measure of comparison for fuzzy sets. Later more measures for comparing fuzzy sets have been introduced (see, among many others ${ }^{2-9}$ ). There also methods of generating dissimilarities from a given fuzzy equivalence relation, by residuation, automorphism, t-norm or conorm, studied by $\mathrm{He}, \mathrm{Li}$ and Qin ( $\left.\mathrm{se}^{10-12}\right)$. A comprehensive study of this topic can be found in Couso et at. ${ }^{13}$ Among all them, the most usual measures of comparison are dissimilarities $\left(\operatorname{see}^{14}\right)$.

The mapping of our interest are interval-valued fuzzy sets, i.e. mappings from the universe $X$ to the set of all closed subintervals of $[0,1]$. Here we face a problem that cannot be presented when working with fuzzy sets only. Namely, the nature of mutual difference between interval-valued fuzzy sets $A, B$ in Fig. 1 is very different from that between interval-valued fuzzy sets $C, D$ in the same figure.

We can think of particular representatives of our interval-valued fuzzy sets in the following way: by a representative of an interval-valued fuzzy set $A$ we understand a fuzzy set $f$ such that $f(x) \in A(x)$ for each $x \in X$. For a given measure of similarity for fuzzy sets and interval-valued fuzzy sets $A, B$ we are able to work with the set of those measures taking all possible pairs of the representatives of $A$ and $B$. However, as we see in the Fig. 1, some representatives of one interval-value fuzzy set may also be representatives of the other one, so we should take into consideration the least and the biggest possible distance of the representatives. This leads us to the idea of expressing the dissimilarity of interval-valued fuzzy sets by means of pairs of non-negative numbers.

Comparing two interval-valued sets we can distinguish two different aspects. First, how far are they in some sense from each other adn second, how "wide" they are. It is obvious also from the Fig. 1, that a single number cannot represent both types of the difference. Our definition provides a more complete information and we suppose it can be useful in some cases where both aspect of the difference are important.

This attitude allows us to present not only the mutual distance of the intervalvalued fuzzy sets, but it also accounts the width of particular values (intervals), therefore we use the term uncertainty-aware similarity measures for such functions. On the other hand, this way we estimate bounds for all reasonable dissimilarity 
measures for a given pair of interval-valued fuzzy sets.

This contribution is organized as follows. In Section 2 we give a short overview of dissimilarities and interval-valued fuzzy sets. In Section 3 we introduce dissimilarity measures between interval-valued fuzzy sets such that the value is an interval and we study their relationship with the well-known dissimilarity measures between fuzzy sets. Then, in Section 4 we show how these new dissimilarities can be applied in a practical situation. Finally, our last section details our conclusions and we discuss possible future lines of research.

\section{Basic notions}

Interval-valued fuzzy sets were introduced independently in the seventies by Grattan-Guiness, ${ }^{15}$ Jahn, ${ }^{16}$ Sambuc $^{17}$ and Zadeh. ${ }^{18}$ From them, a lot of studies have been done about them, and nowadays they are well-known. The same happens with the notions of fuzzy set and dissimilarity measure. Thus, we are going only to introduce the necessary concepts, just to fix the notation along this work.

As fuzzy sets can be considered as a particular case of interval-valued fuzzy sets, we briefly recall its definition and the concept of dissimilarity between them. Let $X$ denote the universe. A fuzzy subset of $X$ is a mapping A from $X$ into the real interval $[0,1]$, where the value $A(x)$, for a particular $x \in X$, represents the degree of membership of $x$ to $A$. For any universe $X, \mathcal{F}(X)$ will denote the set of all fuzzy sets on $X$.

The most usual way to compare two fuzzy sets is by means of similarity measures or their dual measures, the dissimilarity ones. The concept of dissimilarity on $\mathcal{F}(X)$ is given as follows.

Definition 1. A mapping $d: \mathcal{F}(X) \times \mathcal{F}(X) \rightarrow[0,1]$ is said to be a dissimilarity measure between fuzzy sets if it satisfies the following conditions for all $f, g \in \mathcal{F}(X)$ :

(d1) $d(f, g)=0 \Leftrightarrow f=g$,

(d2) $d(f, g)=d(f, g)$,

(d3) if $f \leq g \leq h$ then $d(f, g) \leq d(f, h)$ and $d(g, h) \leq d(f, h)$.

Example 1. Typical examples of dissimilarities are:

- The trivial dissimilarity:

$$
d_{1}(f, g)= \begin{cases}1 & \text { if } f \neq g \\ 0 & \text { if } f=g\end{cases}
$$

- The weighted Hamming distance:

$$
d_{2}(f, g)=\sum_{x \in X} \alpha_{x}|f(x)-g(x)|
$$

where $\alpha_{x} \geq 0$ for any $x \in X$ and $\sum_{x \in X} \alpha_{x}=1$. 
4 Torres-Manzanera, E., Král, P., Janǐs, V., Montes, S.

Fuzzy sets model uncertainty about the membership of an element to a set, but it may appear paradoxical that the membership value itself should be one precise real number. Thus, in order to try to solve this drawback, interval-valued fuzzy sets appeared as a generalisation where the membership value is replaced by a more sophisticated mathematical entity, such as an interval. Therefore, the interval $[0,1]$ is replaced by the set of subintervals of $[0,1]$. Thus, if $C l[0,1]$ denotes the set of all closed subintervals of $[0,1]$, that is, $C l[0,1]=\{[a, b]: a, b \in[0,1], a \leq b\}$, an interval-valued fuzzy set, IVF-set for short, is a mapping $A: X \rightarrow C l[0,1]$. From now on, we will denote the set of all interval-valued fuzzy sets on $X$ as $\mathcal{I V F}(X)$.

As we will deal with closed subintervals of $[0,1]$, we introduce the usual partial $\operatorname{order}^{19,20}$ among them: for any $[a, b],[c, d] \in C l[0,1]$, the inequality $[a, b] \leq[c, d]$ means that $a \leq c$ and $b \leq d$. From here, we can consider a partial order on $\mathcal{I} \mathcal{V} \mathcal{F}(X)$, so the notation $A \leq B$ for any $A, B \in \mathcal{I V \mathcal { F }}(X)$ means that $A(x) \leq B(x)$ for each $x \in X$.

In order to handle different mutual relations of IVF-sets, as we discussed in Section 1, we consider a another type of relation on $\mathcal{I V \mathcal { F }}(X) \times \mathcal{I} \mathcal{V} \mathcal{F}(X)$, which is, in fact, the typical inclusion between sets.

Definition 2. Let $A, B \in \mathcal{I V \mathcal { F }}(X), A(x)=\left[a_{1}(x), a_{2}(x)\right], B(x)=\left[b_{1}(x), b_{2}(x)\right]$ for each $x \in X$. Then $A \sqsubseteq B$ if $b_{1}(x) \leq a_{1}(x) \leq a_{2}(x) \leq b_{2}(x)$ for each $x \in X$.

This relation will be used in Definition 3 for dissimilarity of interval-valued fuzzy sets.

It is immediate that $A \sqsubseteq B$ iff $A(x) \subseteq B(x)$ for each $x \in X$ and that $\sqsubseteq$ is also a partial order on $\mathcal{I} \mathcal{V F}(X)$. Moreover, if $A \sqsubseteq B$ and $B \in \mathcal{F}(X)$, then $A=B$, so this order is just the equality when we deal with fuzzy sets.

Example 2. Let $X=\{x, y\}$ be the universe and let $A, B, C$ be three IVF-sets defined as follows:

\begin{tabular}{|c|c|c|}
\cline { 2 - 3 } \multicolumn{1}{c|}{$x$} & $y$ \\
\hline$A$ & {$[0.2,0.4]$} & {$[0.3,0.7]$} \\
$B$ & {$[0.3,0.5]$} & {$[0.3,0.9]$} \\
$C$ & {$[0.1,0.4]$} & {$[0.2,0.85]$} \\
\hline
\end{tabular}

It is cleat that $A \leq B$ and $A \sqsubseteq C$.

The utility of this kind of sets in order to deal with imprecision is evident. The necessity to compare two sets is a very usual step in many cases. Thus, we will devote next section to study how to adapt the concept of dissimilarity for this kind of sets. The imprecision will be collected by the dissimilarity, since its value will also be an interval.

\section{Dissimilarity on $\mathcal{I V \mathcal { F }}(X)$}

The motivation for our research is the following: Let $d$ be a given dissimilarity on $\mathcal{I V \mathcal { F }}(X)$ and let $A, B$ be two elements in $\mathcal{I V \mathcal { F }}(X)$. Denote by $R_{A}, R_{B}$ the set of 
all representatives for $A, B$ respectively, as described in Section 1. Then the natural bounds for a measure of dissimilarity for $A, B$ should be the numbers $l(A, B)=$ $\inf \left\{d\left(f_{A}, f_{B}\right), f_{A} \in R_{A}, f_{B} \in R_{B}\right\}$ and $u(A, B)=\sup \left\{d\left(f_{A}, f_{B}\right), f_{A} \in R_{A}, f_{B} \in\right.$ $\left.R_{B}\right\}$. Thus we obtain the interval $[l(A, B), u(A, B)]$ spanning from the least possible dissimilarity of the representatives to the largest one. This construction should lead to the axiomatic definition for the dissimilarity of IVF-sets. Based on this motivation we have the following definition, where the difference between two interval-valued fuzzy sets is given by an interval instead of the usual case considered in the literature, where it is just a number. ${ }^{21-25}$ Thus, in the literature, we can find dissimilarity measures between interval-valued fuzzy sets, which are characterized in all the cases by Definition 2 and its dual measures, the similarities. In general, a similarity is obtained from a dissimilarity by applying on it a decreasing map $\varphi$ such that $\varphi(0)=$ 1. Since interval-valued fuzzy-sets are mathematically equivalent to intuitionistic fuzzy sets, a revision about this topic can be found in Montes et al. ${ }^{26}$ Similarities and dissimilarities assume values on the unit interval $[0,1]$. Therefore, the classical definition of dissimilarity cannot be compared to the new one we are going to introduce, since they are working on a different codomain.

Definition 3. The mapping dis : $(\mathcal{I V \mathcal { F }}(X))^{2} \rightarrow C l[0,1]$ is an uncertainty aware dissimilarity measure on $\mathcal{I} \mathcal{V F}(X)$, if for any $A, B, C, D \in \mathcal{I} \mathcal{V F}(X)$ the following conditions are fulfilled:

$(\operatorname{dis} 1) \operatorname{dis}(A, B)=[0,0] \Leftrightarrow A, B \in \mathcal{F}(X), A=B$,

(dis2) $\operatorname{dis}(A, B)=\operatorname{dis}(B, A)$,

(dis3) if $A \leq B \leq C$ then $\operatorname{dis}(A, B) \leq \operatorname{dis}(A, C)$ and $\operatorname{dis}(B, C) \leq \operatorname{dis}(A, C)$,

(dis4) if $A \sqsubseteq B, C \sqsubseteq D$ then $\operatorname{dis}(A, C) \subseteq \operatorname{dis}(B, D)$.

If no confusion can arise, we will speak shortly just on a dissimilarity on $\mathcal{I} \mathcal{V} \mathcal{F}(X)$.

As we can see, the main difference between Definition 2 and Definition 3 is Axiom (dis4). Its aim is to guarantee the connection between the dissimilarity values and inclusion relations of the considered interval-valued sets. In fact thanks to (dis4) the dissimilarity is monotone with respect to a set inclusion.

We try to collect the imprecision we have about the real value of the membership function in the case of interval-valued fuzzy sets. Thus, if the hesitance is lower for set $A$ than for set $B$, the width of the interval obtained when we compare them with a third set $C$ is also lower, since we have more information. This main difference is also realized by the case we compare a set and itself. As the membership value is an interval for any element in $X$, we are comparing interval-valued structures and therefore, two different point membership valued could be allowed. Thus, for arbitrary $A \in \mathcal{I V \mathcal { F }}(X)$ the value $\operatorname{dis}(A, A)$ is of the type $[0, a]$. To show this, take an arbitrary fuzzy set $f_{A} \in R_{A}$. Then $f_{A} \sqsubseteq A$ and from (dis1) and (dis4) we have that $[0,0]=\operatorname{dis}\left(f_{A}, f_{A}\right) \subseteq \operatorname{dis}(A, A)$, which implies that $0 \in \operatorname{dis}(A, A)$. However, the upper bound could be different from zero, as we will see at the next examples. 
6 Torres-Manzanera, E., Král, P., Janǐs, V., Montes, S.

Example 3. An easy example of dissimilarity for interval-valued fuzzy sets is:

$$
\operatorname{dis}_{0}(A, B)= \begin{cases}{[0,0]} & \text { if } A, B \in \mathcal{F}(X), A=B \\ {[0,1]} & \text { otherwise }\end{cases}
$$

It is easy to prove that $d i s_{0}$ is a dissimilarity on $\mathcal{I V F}(X)$ and it can be called the trivial dissimilarity.

If we replace $[0,1]$ by $[1,1]$, the remaining map $d i s_{0}^{*}$ is not a dissimilarity, since for $X=\{x\}, A(x)=C(x)=[0.4,0.4], B(x)=D(x)=[0.2,0.6]$, we have that $A \sqsubseteq B, C \sqsubseteq D$, but dis $s_{0}^{*}(A, C)=[0,0] \nsubseteq[1,1]=\operatorname{dis}_{0}^{*}(B, D)$.

Moreover, we have that $\operatorname{dis}_{0}(B, B)=[0,1]$. Thus, as we commented previously, the upper bound is not always equal to zero.

In the following we clarify the connection between a dissimilarity on $\mathcal{F}(X)$ and the related induced dissimilarity on $\mathcal{I V F}(X)$.

Proposition 1. Let $d$ be a dissimilarity on $\mathcal{F}(X)$. If we consider the mapping dis $_{d}:(\mathcal{I V F}(X))^{2} \rightarrow C l[0,1]$ such that dis $s_{d}(A, B)=[l(A, B), u(A, B)]$, then dis $s_{d}$ is a dissimilarity on $\operatorname{IVF}(X)$, which is said to be induced by $d$.

Proof. (dis1): Let $A, B \in \mathcal{I V F}(X)$, suppose that $\operatorname{dis}_{d}(A, B)=[0,0]$. Then $l(A, B)=u(A, B)=0$, and from the definition of $l$ and $u$ we see that the set $\left\{d\left(f_{A}, f_{B}\right), f_{A} \in R_{A}, f_{B} \in R_{B}\right\}$ is a singleton $\{0\}$. From the property (d1) we have, that $A$ and $B$ have only one representative, hence they belong to $\mathcal{F}(X)$, moreover, there representatives are equal, and so $A=B$.

The converse implication is obvious.

(dis2): This property follows directly from (d2).

(dis3): Let $A, B, C \in \mathcal{I V \mathcal { F }}(X), A \leq B \leq C$. We will show that $\operatorname{dis}_{d}(A, B) \leq$ $\operatorname{dis}_{d}(A, C)$.

Fix an arbitrary $x \in X$. Then $A(x) \leq B(x) \leq C(x)$ and so for any $f_{B}(x)$ there are $f_{A}(x), f_{C}(x)$ such that $f_{A}(x) \leq f_{B}(x) \leq f_{C}(x)$. From (d3) it follows that for arbitrary $f_{B} \in R_{B}$ there are $f_{A} \in R_{A}, f_{C} \in R_{C}$ such that $d\left(f_{A}, f_{B}\right) \leq d\left(f_{A}, f_{C}\right)$. Then we can also claim that

$$
\inf \left\{d\left(f_{A}, f_{B}\right), f_{A} \in R_{A}, f_{B} \in R_{B}\right\} \leq \inf \left\{d\left(f_{A}, f_{C}\right), f_{A} \in R_{A}, f_{C} \in R_{C}\right\} .
$$

In a similar way we can obtain the inequality

$$
\sup \left\{d\left(f_{A}, f_{B}\right), f_{A} \in R_{A}, f_{B} \in R_{B}\right\} \leq \sup \left\{d\left(f_{A}, f_{C}\right), f_{A} \in R_{A}, f_{C} \in R_{C}\right\} .
$$

From both these we obtain $\operatorname{dis}_{d}(A, B) \leq d i s_{d}(A, C)$. By the same argumentation we can show the inequality $\operatorname{dis}_{d}(B, C) \leq \operatorname{dis}_{d}(A, C)$.

(dis4): Let $A, B, C, D \in \mathcal{I} \mathcal{V F}(X), A \sqsubseteq B, C \sqsubseteq D$. Then $R_{A} \subseteq R_{B}, R_{C} \subseteq R_{D}$ and so we have

$$
\left\{d\left(f_{A}, f_{C}\right), f_{A} \in R_{A}, f_{C} \in R_{C}\right\} \subseteq\left\{d\left(f_{B}, f_{D}\right), f_{B} \in R_{B}, f_{D} \in R_{D}\right\} .
$$

The required property $\operatorname{dis}_{d}(A, C) \subseteq \operatorname{dis}_{d}(B, D)$ is a direct consequence of this inclusion. 
Thus, we can consider the induced dissimilarities from the examples considered in Example 1.

\section{Example 4.}

- From the trivial dissimilarity $d_{1}$ we obtain the dissimilarity for interval-valued fuzzy sets given by

$$
\operatorname{dis}_{1}(A, B)=\left\{\begin{array}{l}
{[0,0] \text { if } f_{A}=f_{B}, \forall f_{A} \in R_{A}, f_{B} \in R_{B}} \\
{[0,1] \text { if } A \neq B \text { but } \exists f_{A} \in R_{A}, f_{B} \in R_{B} \text { with } f_{A}=f_{B}} \\
{[1,1] \text { if } f_{A} \neq f_{B}, \forall f_{A} \in R_{A}, f_{B} \in R_{B}}
\end{array}\right.
$$

Clearly, the condition $f_{A}=f_{B}, \forall f_{A} \in R_{A}, f_{B} \in R_{B}$ is equivalent to say that $A$ and $B$ are two equal fuzzy sets.

- From the weighted Hamming distance between fuzzy sets $d_{2}$ we obtain the dissimilarity between interval-valued fuzzy sets $d i s_{2}$ defined as

$$
\left[\inf _{f_{A} \in R_{A}, f_{B} \in R_{B}} \sum \alpha_{x}\left|f_{A}(x)-f_{B}(x)\right|, \sup _{f_{A} \in R_{A}, f_{B} \in R_{B}} \sum_{x} \alpha_{x}\left|f_{A}(x)-f_{B}(x)\right|\right]
$$

for any $A, B \in \mathcal{I} \mathcal{V} \mathcal{F}(X)$, where $\alpha_{x} \geq 0, \forall x \in X$ and $\sum_{x} \alpha_{x}=1$.

If we consider the interval-valued fuzzy sets introduced in Example 2, we have that $\operatorname{dis}_{1}(A, B)=[0,1]$ and $\operatorname{dis}_{2}(A, B)=[0,0.45]$ if $\alpha_{x}=\alpha_{y}=1 / 2$.

The order of dissimilarities on $\mathcal{F}(X)$ is inherited for the induced ones:

Proposition 2. Let $d_{1}, d_{2}$ be two dissimilarities on $\mathcal{F}(X)$ such that $d_{1} \leq d_{2}$. Then for each $A, B \in \mathcal{I V \mathcal { F }}(X)$ there is $\operatorname{dis}_{d_{1}}(A, B) \leq \operatorname{dis}_{d_{2}}(A, B)$.

The proof follows directly from the respective definitions.

Since the starting point is a dissimilarity between fuzzy sets, the induced dissimilarity between fuzzy sets should match with the initial one when fuzzy sets are compared. This follows immediately from the identities $R_{f}=\{f\}, R_{g}=\{g\}$ for any $f, g \in \mathcal{F}(X)$. Thus,

Corollary 1. If dis $s_{d}$ is induced by $d$, then for any $f, g \in \mathcal{F}(X)$ there is dis $s_{d}(f, g)=$ $[d(f, g), d(f, g)]$.

We have shown a method how any dissimilarity on $\mathcal{F}(X)$ generates a dissimilarity on $\mathcal{I} \mathcal{V} \mathcal{F}(X)$, moreover, as for all $f, g \in \mathcal{F}(X)$ we have $\operatorname{dis}_{d}(f, g)=$ $[d(f, g), d(f, g)]$, the mapping $d \mapsto d i s_{d}$ is injective. A natural question is, whether it is even a bijection, i.e. whether any dissimilarity on $\mathcal{I V F}(X)$ is generated by some dissimilarity on $\mathcal{F}(X)$. The following example shows that the answer to this question is negative.

Example 5. Let's consider again the dissimilarity $d i s_{0}$ on $\mathcal{I V F}(C)$ introduced in Example 3. If there exists a dissimilarity $d$ on $\mathcal{F}(X)$ such that $d i s_{d}=d i s_{0}$, then 
8 Torres-Manzanera, E., Král', P., Janiš, V., Montes, S.

from the previous corollary, we know that $d i s_{0}(f, g)=[d(f, g), d(f, g)]$ for any pair of fuzzy sets $f$ and $g$. But if we consider $f \neq g$, we have that $\operatorname{dis}_{0}(f, g)=[0,1]$ and therefore $d i s_{0}$ cannot be induced by any other dissimilarity on $\mathcal{F}(X)$.

The restriction for fuzzy sets considered in Corollary 1 can be done for any dissimilarity between interval-valued fuzzy sets, not only for the ones induces by dissimilarities between fuzzy sets. Thus, if we consider that $[x, y]_{2}$ denotes the second component of the pair $[x, y]$, we have that

Proposition 3. Let dis be a dissimilarity on $\operatorname{IVF}(X)$. The map $d_{\text {dis }}: \mathcal{F}(X) \times$ $\mathcal{F}(X) \rightarrow[0,1]$ defined as $d_{\text {dis }}(f, g)=[\operatorname{dis}(f, g)]_{2}$ for any $f, g \in \mathcal{F}(X)$ is a dissimilarity on $\mathcal{F}(X)$.

Proof. (d1): Let $f, g \in \mathcal{F}(X)$. Then $d_{d i s}(f, g)=0$ is equivalent to $[\operatorname{dis}(f, g)]_{2}=0$, what is the same as $\operatorname{dis}(f, g)=[0,0]$. By (dis1) this gives $f=g$.

(d2): The symmetry follows directly from the symmetry of dis.

(d3): Let $f, g, h \in(F(X), f \leq g \leq h$. Then by (dis3) there is $\operatorname{dis}(f, g) \leq \operatorname{dis}(f, h)$ and from the definition of the $\leq$ relation in $C l[0,1]$ we have $[\operatorname{dis}(f, g)]_{2} \leq[\operatorname{dis}(f, h)]_{2}$ and therefore $d_{d i s}(f, g) \leq d_{d i s}(f, h)$. The inequality $d_{d i s}(g, h) \leq d_{d i s}(f, h)$ can be shown analogically.

It is clear from Proposition 3 that if $d i s_{1} \leq d i s_{2}$ then $d_{d i s_{1}} \leq d_{d i s_{2}}$, that is, the order of dissimilarities on $\mathcal{I V F}(X)$ is also inherited for the associated dissimilarities on $\mathcal{F}(X)$.

Example 6. If we consider the dissimilarities between interval-valued fuzzy sets given in Examples 3 and 4, we obtain that $d_{d i s_{0}}=d_{d i s_{1}}$ and they coincide with the trivial dissimilarity between fuzzy sets $d_{1}$ and that $d_{d i s_{2}}$ coincides with the weighted Hamming distance between fuzzy sets.

Moreover, since $d_{d i s_{0}}=d_{d i s_{1}}$, we have proven that the mapping dis $\mapsto d_{d i s}$ is not injective.

Constructing dissimilarities we can start with $d$ on $\mathcal{F}(X)$, then create the induced dissimilarity on $\mathcal{I V F}(X)$ and that restrict it to $\mathcal{F}(X)$. At the previous examples we could see that we return back to the $d$. Is this true in general? The answer is given at the next proposition.

Proposition 4. Let d be a dissimilarity on $\mathcal{F}(X)$. If we obtain the induced dissimilarity dis $_{d}$ on $\mathcal{I V \mathcal { F }}(X)$ and we use Proposition 3 to obtain the dissimilarity $d_{d i s_{d}}$ on $\mathcal{F}(X)$, then $d_{d i s_{d}}=d$.

Proof. Note first that in case $f, g \in \mathcal{F}(x)$ there is $R_{f}=\{f\}$ and $R_{g}=\{g\}$. Because of this we have $l(f, g)=u(f, g)=d(f, g)$. By use of these facts we obtain

$$
d_{d i s_{d}}(f, g)=\left[d i s_{d}(f, g)\right]_{2}=u(f, g)=d(f, g)
$$

and the identity is proved. 
Thus, if we consider the class of dissimilarities on $\mathcal{F}(X)$ and the class of dissimilarities on $\mathcal{I} \mathcal{V} \mathcal{F}(X)$ induced by fuzzy dissimilarities, both classes are isomorphic. However, not all the dissimilarities on $\mathcal{I V} \mathcal{F}(X)$ are induced by fuzzy dissimilarities, as we could see in Example 5. Thus, the set of dissimilarities on $\mathcal{I V} \mathcal{F}(X)$ is substantially richer than that on $\mathcal{F}(X)$.

Once we have studied in deep the relationship between the concept of uncertainty aware dissimilarity measure and the well-known dissimilarity measures between fuzzy sets, we could think on a theoretical study of the properties fulfilled by this new concept. However, as we could see in several previous studies, ${ }^{4,5,27}$ even in the case of numerical measures, an extra requirement has to be considered in order to obtain interesting properties. This is the property of the locality, which is based on the pointwise decomposition of the measure for any element of the universe. For this decomposition, an addition or in general an aggregation function has to be considered. Thus, when we are working with intervals, this is not so straight and a deep and detailed study should be considered, with some previous analysis about the appropriate way to aggregate the intervals.

\section{Example of application}

Dissimilarities discussed in the previous sections are applied in analysis of lake deposits of the High Tatra Mountains within the project Deglaciation and postglacial climatic evolution recorded in the lake deposits of the High Tatra Mountains (APVV-15-0292). As a part of the research project, cyclicities in sedimentary records are studied using time series data extracted from drilled sedimentary cores. These cores are taken from the bottom of the selected High Tatra Mountains lakes, vertically split, and scanned. The resulting core images display climatic changes as gray stripes of different intensity. The stripes we get this way are then transformed into a time series for analysis using some standard methods like spectral analysis, etc.

Unfortunately, quite often, the cores have some deformities, e.g. some parts are partially shifted to each other or missing which makes transformation to figures difficult and error prone. In order to minimize the impact of image imperfections on the whole transformation process, we decided to include an image quality measurement based on dissimilarities in it. Our idea stems from the fact that each stripe can be represented by a triple (rank, start point, endpoint) and this representation can be interpreted as an interval-valued fuzzy set $A$, for which $A($ rank $)=$ [startpoint, endpoint]. For each vertically aligned core image, present stripes are identified independently in its left and right side and matched by their ranks. Then local dissimilarities between matching left and right stripes are computed.

Finally, a dissimilarity between complete left and right stripe representations is determined. If dissimilarity between the left and right stripe representations is unsatisfactory or if some local dissimilarities are too high, matching stripes with 
problematic values of dissimilarities are closely inspected and, if possible, their transformation corrected. The analysis is then conducted separately on the dissimilarity corrected left (right) stripe representation as well as on the average of left and right representations.

We illustrate the above mentioned application numerically using the following example.

Example 7. For simplicity, let us assume that we are measuring five consecutive time points in a piece of core image. The length of that piece is ten centimetres and bounds for each stripe position are computed as a distance in centimetres of the corresponding bound from the beginning of the core image divided by the length of the image. In this scenario, let us assume that we got the following interval-valued fuzzy sets representing the stripes.

$$
\begin{aligned}
& L=(1 /[0.1,0.1], 2 /[0.15,0.16], 3 /[0.17,0.19], 4 /[0.21,0.22], 5 /[0.23,24]) \\
& R=(1 /[0.1,0.1], 2 /[0.14,0.16], 3 /[0.17,0.20], 4 /[0.23,0.24], 5 /[0.23,24])
\end{aligned}
$$

Let use "rough" dissimilarities $d i s_{0}$ and $d i s_{1}$ from Examples 3 and 4, respectively. We use $d i s_{0}$ to compute a "global" dissimilarity between the left and right stripes and $d i s_{1}$ to get an interval-valued fuzzy set representing "local" dissimilarities (point by point):

$$
\begin{gathered}
\operatorname{dis}_{0}(L, R)=[0,1] \\
\text { localdis }_{1}(L, R)=(1 /[0,0], 2 /[0,1], 3 /[0,1], 4 /[1,1], 5 /[0,1]) .
\end{gathered}
$$

Interpretation of the result of this type would be that differences between stripes are too high. The most problematic measurement is present in the forth time point, where the local dissimilarity equals to $[1,1]$.

\section{Conclusions}

In this work we have introduced a new method for the comparison of two intervalvalued fuzzy sets, where the value of the difference between them is an interval instead of a number. The proposed method is a generalization of the classical dissimilarities between fuzzy sets. It deals with the imprecision by obtaining an imprecise value of the difference of two imprecise sets.

We have also investigated some relationships among dissimilarities between fuzzy sets and dissimilarities between interval-valued fuzzy sets. We have showed that the interval-valued dissimilarities allow us to do comparisons taking into account more points of view. This new method could be an appropriate alternative in some real applications in which the data are interval-valued. An example where these measures are applied is also explained. 


\section{Acknowledgements}

The second author would like to express his thank to the support provided by Science and Technology Assistance Agency (APVV) under contract No. APVV-150292. He also would like to express his thank to the support provided by Slovak Grant Agency VEGA, grant 1/0093/17. The research reported in this paper by the first and fourth authors has been supported by FICYT Project IDI/2018/000176 and Project PGC2018-098623-B-I00.

\section{References}

1. B. Bouchon-Meunier, M. Rifqi and S. Bothorel, "Towards general measures of comparison of objects", Fuzzy Sets and Systems 84 (1996) 143-153.

2. M. Anthony and P. L. Hammer, "A boolean measure of similarity", Discrete Applied Mathematics 154 (2006) 2242-2246.

3. I. Couso and S. Montes, "An axiomatic definition of fuzzy divergence measures", International Journal Of Uncertainty Fuzziness and Knowledge-based Systems 16.

4. V. Kobza, V. Janis and S. Montes, "Generalizated local divergence measures", Journal of Intelligent and Fuzzy Systems 33 (2017) 337-350.

5. S. Montes, I. Couso, P. Gil and C. Bertoluzza, "Divergence measure between fuzzy sets", International Journal of Approximate Reasoning 30 (2002) 91-105.

6. L. Valverde and S. Ovchinnikov, "Representations of t-similarity relations", Fuzzy Sets and Systems 159 (2008) 2211-220.

7. A. Wilbik and J. M. Keller, "A fuzzy measure similarity between sets of linguistic summaries", IEEE Transactions on Fuzzy Systems 21 (2013) 183-189.

8. L. Zadeh, "A note on similarity-based definitions of possibility and probability", Information Sciences 267 (2014) 334-336.

9. C. Zhang and H. Fu, "Similarity measures on three kinds of fuzzy sets", Pattern Recognition Letters 27 (2006) 1307-1317.

10. X. He, Y. Li and K. Qin, "Distance measures on intuitionistic fuzzy sets based on intuitionistic fuzzy dissimilarity functions", Soft Computing (2019) 1-19.

11. Y. Li, K. Qin and X. He, "Some new approaches to constructing similarity measures", Fuzzy Sets and Systems 234 (2014) 46-60.

12. Y. Li, K. Qin and X. He, "Dissimilarity functions and divergence measures between fuzzy sets", Information Sciences 288 (2014) 15-26.

13. I. Couso, L. Garrido and L. Sánchez, "Similarity and dissimilarity measures between fuzzy sets: A formal relational study", Information Sciences 229 (2013) 122-141.

14. X. Lui, "Entropy, distance measure and similarity measure of fuzzy sets and their relations", Fuzzy Sets and Systems 52 (1992) 305-318.

15. I. Grattan-Guiness, "Fuzzy membership mapped onto interval and many-valued quantities", Z. Math. Logik. Grundladen Math 22 (1975) 149-160.

16. K. Jahn, "Intervall-wertige mengen", Mathematische Nachrichten 33 (2017) 337-350.

17. R. Sambuc, "Fonctions $\phi$-floues. application l'aide au diagnostic en pathologie thyroidienne", Ph.D. thesis, University Marseille, France, 1975.

18. L. Zadeh, "The concept of a linguistic variable and its application to approximate reasoning-i", Information Sciences 8 (1975) 199-249.

19. H. Bustince, J. Fernandez, A. Kolesarova and R. Mesiar, "Generation of linear orders for intervals by means of aggregation functions", Fuzzy Sets and Systems 220 (2013) 69-77. 
12 Torres-Manzanera, E., Král', P., Janǐ̌, V., Montes, S.

20. R. Perez-Fernandez, P. Alonso, H. Bustince, I. Diaz, A. Jurio and S. Montes, "Ordering finitely generated sets and finite interval-valued hesitant fuzzy sets", Information Sciences 325 (2015) 375-392.

21. M. Arefi and S. Taheri, "Weighted similarity measure on interval-valued fuzzy sets and its application to pattern recognition", Iranian Journal of Fuzzy Sets 11 (2014) $67-79$.

22. G. Deng, L. Song, Y. Jiang and J. Fu, "Monotonic similarity measures of intervalvalued fuzzy sets and their applications", International Journal of Uncertainty, Fuzziness and Knowledge-Based Systems 25 (2017) 515-544.

23. P. Grzegorzewski, "Distances between intuitionistic fuzzy sets and/or interval-valued fuzzy sets based on the hausdorff metric", Fuzzy Sets and Systems 148 (2004) 319-328.

24. W. Zeng and P. Guo, "Normalized distance, similarity measure, inclusion measure and entropy of interval-valued fuzzy sets and their relationship", Information Sciences $\mathbf{1 7 8}$ (2008) 1334-1342.

25. H. Zhang, W. Zhang and C. Mei, "Entropy of interval-valued fuzzy sets based on distance and its relationship with similarity measure", Knowledge-Based Systems 22 (2009) 449-454.

26. I. Montes, N. Pal, V. Janis and S. Montes, "Divergence measures for intuitionistic fuzzy sets", IEEE Transactions on Fuzzy Systems 23 (2015) 444-456.

27. I. Montes, V. Janis, N. Pal and S. Montes, "Local divergences for atanassov intuitionistic fuzzy sets", IEEE Transactions on Fuzzy Systems 24 (2016) 360-373. 